\title{
Adolescent Girls Empowerment Program (AGEP): Evaluation-Round 4 update
}

\author{
Karen Austrian \\ Population Council \\ Paul C. Hewett \\ Population Council \\ Erica Soler-Hampejsek \\ Jean Digitale \\ Population Council
}

Follow this and additional works at: https://knowledgecommons.popcouncil.org/departments_sbsr-pgy

Part of the Demography, Population, and Ecology Commons, Family, Life Course, and Society Commons, and the International Public Health Commons How does access to this work benefit you? Let us know!

\section{Recommended Citation}

Austrian, Karen, Paul C. Hewett, Erica Soler-Hampejsek, and Jean Digitale. 2017. "Adolescent Girls Empowerment Program (AGEP): Evaluation-Round 4 update," brief. Lusaka: Population Council. 


\section{ADOLESCENT GIRLS EMPOWERMENT PROGRAM (AGEP): EVALUATION- ROUND 4 UPDATE}

\section{INTRODUCTION}

Social isolation, economic vulnerability, and lack of access to health care and education prevent healthy transitions from childhood to adulthood, especially for adolescent girls in developing countries. In Zambia, poor girls often are at higher risk of gender-based violence, unintended pregnancy, and HIV. Many drop out of school, are unable to find employment, lack the ability to make independent decisions, and are not being reached by existing programs for young people. The root causes of these challenges may reside at the social, community, family, or even at the level of the adolescent girls, whether the reasons are poverty, regressive social and cultural gender norms, or lack of self-esteem on the girls' part. These challenges are interlinked, suggesting that solutions need be multi-sectoral as well.

The Adolescent Girls Empowerment Program (AGEP) was conducted over two-years to support more than 11,000 of the most vulnerable ${ }^{1}$ adolescent girls in Zambia. The AGEP intervention was led by the Population Council, in partnership with the Young Women's Christian Association of Zambia (YWCA), the National Savings and Credit Bank of Zambia (Natsave), and the Government of Zambia. AGEP was based on an asset-building framework that posited that by enhancing girls' social, health, and economic assets in the short term, more positive longer-term dividends would be achieved on health and education outcomes. AGEP was implemented in ten sites within urban and rural areas in four provinces in Zambia. The AGEP intervention was comprised of three major components: 1) weekly safe spaces groups in which girls met over the course of two years for training on sexual and reproductive health, life skills, and financial education; 2) a health voucher that girls could use at contracted private and public facilities for a package of general wellness and sexual and reproductive health services; and, 3) a savings account that was designed specifically to be girl-friendly.

To assess the impact of AGEP on mediating and longer-term demographic, reproductive, and health outcomes, Population Council researchers designed and implemented a longitudinal, cluster randomized controlled trial across all program areas. A baseline survey was conducted in 2013 prior to program implementation and data has been collected annually. A third round of data collection in 2015 produced the midline findings, measuring the program effect immediately at the end of AGEP. Details of the mid-term results were published in a full technical report, executive summary, and brief.

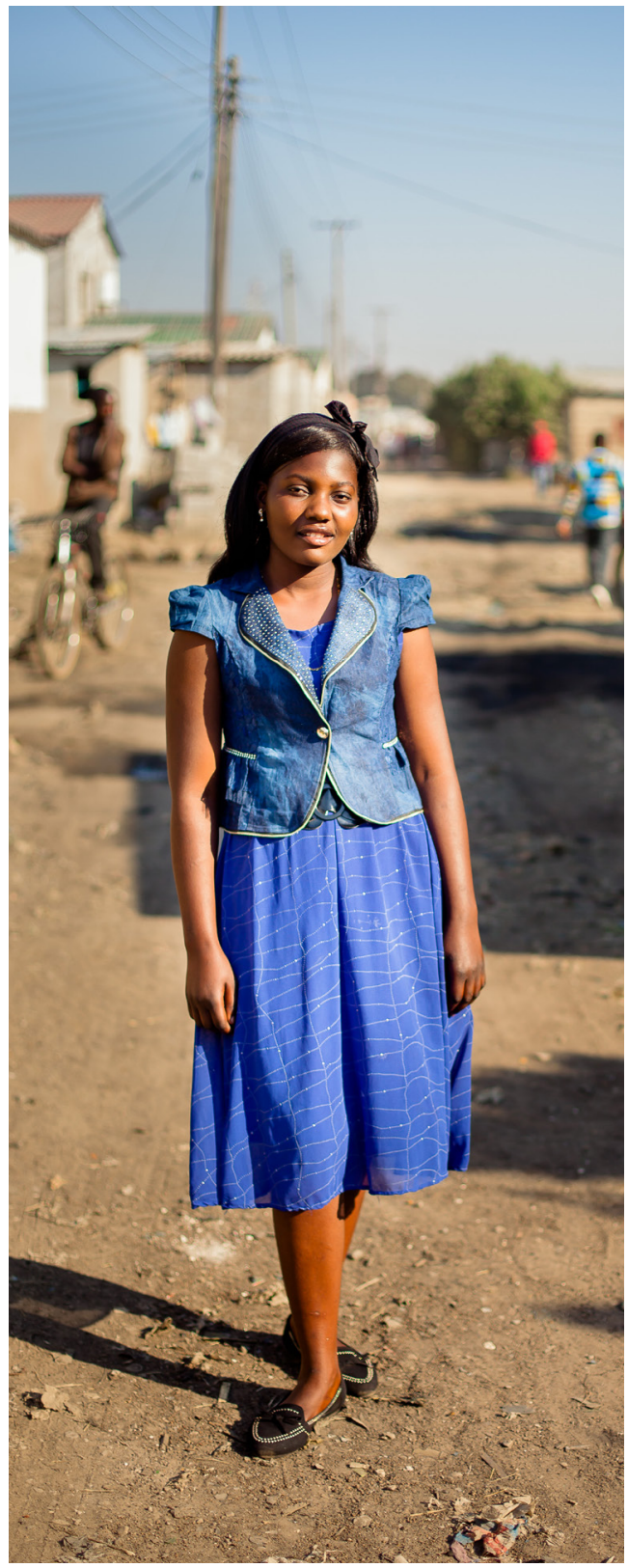

1 In AGEP, "vulnerable" was defined as the number of years of schooling a girl had attained, relative to her age, taking into account several individual and household factors. Please see the brief entitled "Methodology: Reaching the most vulnerable adolescent girls": http://www.popcouncil.org/uploads/pdfs/2016PGY_AGEP-Vulnerability_brief.pdf.

\author{
The Population Council conducts research and delivers solutions that \\ improve lives around the world. Big ideas supported by evidence: \\ It's our model for global change. popcouncil.org \\ (c) 2017 The Population Council, Inc.
}
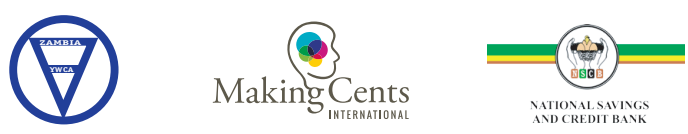

This project was funded by UK aid from the UK government. 
The focus of this brief is to provide an update on the 2015 mid-term findings using the fourth round of data collection completed in December 2016. The results from the Round 4 data represent the experiences and outcomes of adolescent girls one year after the program ended.

\section{ROUND 4 FINDINGS}

At the end of the program in 2015, AGEP girls scored almost one point higher on a sexual and reproductive health knowledge scale $(p<0.05)$ and had greater access to safe places in the community apart from home and school (DID coef $5.6 \%$; $p$ 0.05). A year later in 2016, AGEP girls continued to have significantly higher levels of SRH knowledge, but with the closing of the weekly girls' groups they reported that they no longer had as much access to safe places in the community, suggesting the communities were unable to sustain a dedicated space for girls. Additionally, while AGEP girls had improved financial literacy by one point on a nine-point scale $(p<0.05)$ and savings (DID coef $17.5 \%$; $p<0.01$ ) relative to the control sample in the mid-term results, there was no longer a significant difference between AGEP girls and non-AGEP girls one year after the program, apart from the girls who had access to savings accounts who retained similar results as at midline.

At midline, AGEP girls were ten percentage points less likely to ever have transactional sex $(p<0.05)$ and nine percentage points more likely to use a condom during their first sexual intercourse $(p<0.05)$; both of these impacts remained significant one year after the midline. Similar to the midline findings, there was no change as of yet on longer-term sexual health and behavior outcomes such as sexual initiation, experiences of violence, or rates of unintended pregnancy. However, it is interesting to note that while the AGEP girls in the urban sample were more likely to have ever had sex, the AGEP girls in the rural sample were significantly less likely to have ever had sex relative to the control group.

Additionally, while at midline there was no significant improvement on self-efficacy and acceptance of intimate partner violence, one year after the program the older cohort showed a modest but positive impact in these two areas (self-efficacy: DID coef: 0.303, $p<.10$; acceptability of IPV: DID coef: $8.5 \%, p<0.05)$. One possible explanation is that as girls grow older and are more likely to be entering into longer-term relationships, the lessons learned in the program become more relevant.

Finally, among the younger girls in both urban and rural areas, there were small yet significant increases in the number of years of schooling completed as compared to the girls in the control group (DID coef - 0.12; $p<0.1$ ).
Summary of Round 4 Findings

Mid-term impacts that remained after one year:

- Improved SRH knowledge

- Improved financial literacy (among girls with access to savings accounts)

- Reduced transactional sex

- Increased use of condoms at first sexual intercourse

Mid-term impacts that disappeared after one year:

- Access to safe spaces in the community

- Improved financial literacy (among girls without access to savings accounts)

- Improved savings behavior

New impacts that appear one year after the mid-term:

- Improved self-efficacy (among older adolescent girls)

- Lower acceptance of intimate partner violence (among older adolescent girls)

- Increased number of years of schooling completed (among younger adolescent girls)

\section{CONCLUSION}

The Round 4 update on the findings raises interesting distinctions between which positive impacts are retained and which are lost after program participation ceases. One explanation is that some positive changes need ongoing "reminders" or "nudges" that the program was providing, such as financial literacy skills (unless one has a savings account) and savings behavior, while others can be retained without direct reminders, such as SRH knowledge. Other questions also arise around the possibility of areas of program influence that remain latent until girls age and face direct experiences in which they are able to apply lessons learned from the intervention.

The final round of data collection, to be collected in the second half of 2017, will provide more detail and confirmation on these post-intervention effects, exploring if the trends continue, strengthen, or wash out altogether.

Authors: Karen Austrian, Paul C. Hewett,

Erica Soler-Hampejsek, Jean Digitale

Population Council, Zambia

Plot 3670, No. 4

Mwaleshi Road, Olympia Park

Lusaka, Zambia

Tel: +260 211295925

email:info.zambia@popcouncil.org

Photo: John Healey 